\title{
Review
}

\section{An overview on ethical issues about sperm donation}

\author{
Dan Gong ${ }^{1,2}$, Yu-Lin Liu ${ }^{1}$, Zhong Zheng ${ }^{1}$, Yi-Fei Tian ${ }^{3}$, Zheng Li $^{1}$ \\ ${ }^{1}$ Renji Hospital, Shanghai Human Sperm Bank, Shanghai Institute of Andrology, Shanghai Jiaotong University School of \\ Medicine, Shanghai 200001, China \\ ${ }^{2}$ Harvard College, Harvard University, Cambridge, MA 02138, USA \\ ${ }^{3}$ Shanghai Jiao Tong University School of Medicine, Shanghai 200001, China
}

\begin{abstract}
Beyond the scientific progress in assisted reproductive technologies (ART), it is necessary to discuss the ethical considerations behind these advances. Ethical issues concerning sperm donation have been considered and discussed by government and non-governmental agencies, the public, media and academic institutions in many countries. Recommendations and guidelines concerning sperm donation issues vary from country to country and between professional groups within countries. This paper attempts to present an overview of findings and reports from various agencies concerning the ethics of sperm donation. The following topics are considered: limiting the number of donor offspring; minimizing risk of infection and genetics from sperm donors; age requirements for sperm donors; and anonymity versus non-anonymity of sperm donors. The diversity of policies shows that each country has its unique set of guidelines tailored toward its own specific needs. Similarly, countries designing their own procedures and guidelines concerning reproductive medicine must tailor them toward their own needs and practical considerations. In Mainland China, the anonymous policy for sperm donation should still be carried out, and the number of donor offspring should be revaluated. ART procedures must be conducted in a way that is respectful of those involved. Ethical principles must respect the interests and welfare of persons who will be born as well as the health and psychosocial welfare of all participants, including sperm donors.
\end{abstract}

Asian Journal of Andrology (2009) 11: 645-652. doi: 10.1038/aja.2009.61; published online 21 September 2009.

Keywords: ethics, donor, sperm bank, sperm donation

\section{Introduction}

Assisted reproductive technology (ART) has become increasingly popular over the past several decades.

Correspondence to: Prof. Zheng Li, Department of Urology, Shanghai Institute of Andrology, Shanghai Human Sperm Bank, Shanghai Jiao Tong University School of Medicine, Shanghai 200001, China.

Fax: +86-21-6373-2926 E-mail: doc.zheng.li@gmail.com

Received: 4 May 2009

Revised: 27 May 2009

Accepted: 20 August 2009 Published online: 21 September 2009
The advances in human sperm cryopreservation in the past 50 years and the creation of sperm banks have facilitated the increase in artificial insemination with donor sperm (AID) $[1,2]$. In cases of severe male infertility, the use of donor sperm is the only approach to infertility treatment [3]. Although the ethical concern with introducing a third party into the fertilization procedure by means of donor sperm must be considered as controversial, careful counseling and informed consent by all parties related should help to resolve many of the dilemmas. In 2001, the Chinese Ministry of Health established a standard protocol for human 
sperm banking. Currently, there are 11 sperm banks across Mainland China with licenses from the Chinese government. The advances in sperm cryopreservation have created the opportunity for paternity for many Chinese families.

As ART becomes more popular, ethical considerations surrounding the use of these technologies becomes increasingly important. Ethical issues on sperm donations have been widely discussed in literature. European Society of Human Reproduction and Embryology (ESHRE) stated that gamete donation should focus on the issues raised by the meaning of genetic links, regulation and the necessity for taking into account the welfare of the donor children. Relevant specific aspects concern anonymity, compensation for donation, and the consent, screening and assessment of donors and recipients [4]. Much of the ethical considerations pertain on an individual basis to the definition of parental responsibility. Even in countries in which sperm donation guidelines have been well established, surveys of private citizens have been conducted to assess feedback on current ethical guidelines, leading to our understanding that counseling has a beneficial impact on donors [5-7]. Although most guidelines come from governmental, non-governmental or academic institutions, religious organizations can also offer opinions that the public will value. For example, Catholic teaching on infertility treatment and reproductive technology emphasized the ethical need for children to be conceived and born of the marriage union [8]. As the ethical issues are multifaceted and complicated, recommendations and guidelines concerning reproductive issues are variable from country to country, and between professional groups within countries. Most developing countries, including Mainland China, should learn the lessons from developed countries when designing their own guidelines on sperm donation, but they must also take into consideration cultural tradition, public or patients' opinions, opinions of different religions, economy development and population numbers. An attempt looking at international gamete transactions between countries has been done in the past as an attempt to bridge guidelines between different countries [9]. This review overviews findings and reports from various agencies concerning the ethics of sperm donation. Topics of limiting the number of donor offspring; minimizing the risk of infection and genetics from sperm donors; age requirements for sperm donors; anonymity versus non-anonymity of sperm donors are reviewed. We suggest that the ethical issues on recommendation of sperm donation in Mainland China should be updated to match infertile couples' need for AID or ART, the anonymity policy for sperm donation should still be carried out, the number of donor offspring should be revaluated and medical standards for sperm donors should be improved according to WHO (World Health Organization) laboratory manual for the examination of human semen and spermcervical mucus interaction, fifth edition, which will be published in 2009. Ethical principles must respect the interests and welfare of persons who will be born as well as the health and psychosocial welfare of all participants.

\section{Limiting the number of donor offspring}

The importance of limiting the number of donor offspring from a single sperm donor relates to preventing accidental consanguinity between donor offspring. All countries agree that the potential for consanguinity is a problem, but different countries have developed different guidelines for limiting the number of donor offspring. Considerations include the size of the country's population, density of population and mobility of population. For example, in Mainland China, each sperm donor can only impregnate five women through AID or in vitro fertilization (IVF), whereas the American Society for Reproductive Medicine (ASRM) recommends a limit of 25 children per population of 800000 for a single donor.

The International Federation of Gynecology and Obstetrics (FIGO), the key organization that brings together professional societies of obstetricians and gynecologists on a global basis, provides a general guideline on limiting the number of donor offspring. FIGO recommends that the number of donations from any single donor should be limited to avoid the future danger of consanguinity and/or incest [10]. Current standards or recommendations on donor limits by country are shown below.

\subsection{United States}

In the United States, there is no federal or state law limiting sperm donation. ASRM recommends that institutions, clinics and sperm banks should maintain sufficient records to allow a limit to be set for the number of pregnancies for which a given donor is 
responsible. It is difficult to provide a precise number of times that a given donor's sperm can be used because one must take into consideration the population base from which the donor is selected and the geographic area that may be served by the donor. It has been suggested that in a population of 800000 , limiting a single donor to no more than 25 births would avoid any significant increased risk of inadvertent consanguineous conception. This suggestion may require modification if the population using donor insemination represents an isolated subgroup or if the specimens are distributed over a wide geographic area [11].

\subsection{United Kingdom}

The Human Fertilization and Embryology Authority (HFEA) is the UK's independent regulator overseeing the use of gametes and embryos in fertility treatment and research. It requires that gametes (or embryos created using gametes) from an individual donor should not be used to produce children for more than 10 families, as a result of licensed assisted conception services. Notwithstanding the foregoing, gametes (or embryos created using gametes) from an individual donor may be used in any licensed assisted conception treatment for the purpose of producing a genetically related sibling for an existing child of the family of the woman to be treated [12]. Currently, an individual donor may only be used to produce 10 live birth events (with some exceptions). Multiple simultaneous births all count as one "live birth." The most common exception is when there are more than 10 live birth events from a donor to provide genetically related brothers or sisters for children previously born from a donation. In addition, donors may set their own lower limits on the use of their gametes [13]. Although it was noted that the statistical risk of consanguinity would support a limit much higher than the 10 live birth events specified in current HFEA guidelines, concern was expressed about the emotional and psychological effect on donor-conceived people of the knowledge that there may be a large number of half-siblings. Although a higher sperm donor limit would increase the availability of infertility treatment, there was general support for maintaining an upper limit rather than removing it entirely. An HFEA Steering Group proposed that the HFEA's policy be amended so that the limit is calculated in terms of families using a given donor rather than live birth events. This approach was supported, although there was no consensus on the limit to be adopted. Limits of four families (as in New Zealand) and 10 families (the maximum currently possible in the UK) were suggested [14].

\subsection{Australia}

Different regions of Australia have different limitations on the number of donor-conceived children from the same donor depending on population density and sparseness. In Western Australia, under the Human Reproductive Technology Act of 1991, each donor may contribute to a maximum of five recipient families including donations made to families that reside outside Western Australia, unless the council has given specific approval. However, there is no limit to the number of children to be donor-conceived within each family. This limit is, in part, to minimize the risk of genetic disease arising from the inadvertent marriage of halfsiblings in later life, and also to limit the number of families that donor-conceived people would be related to. Feedback from donor-conceived adults suggests that it may be less bewildering to know that you are related to others in up to five other families [15]. In Victoria, current regulations set a maximum of 10 families per donor (that is, there may be more children within the 10 families) [16].

\subsection{Mainland China}

Donor sperm banks also have a crucial role in Mainland China to improve the development of reproductive medicine in China. The Chinese Ministry of Health has published guidelines for screening and testing anonymous donors for sperm donation. In Mainland China, each sperm donor can only impregnate five women through AID or IVF. Sperm banks are required to follow-up with AID or IVF results, and keep its records to limit the number of pregnancies with the same donor. A computer management system is used to record this data. Even though the population in Mainland China has exceeded 1.3 billion, many couples who suffer from serious male infertility are seeking AID or IVF procedures. However, qualified sperm donors are in such limited numbers that infertile couples often wait for long periods before receiving donor sperm. Data from the Department of Reproductive Medicine at Renji Hospital, Shanghai Jiao Tong University School of Medicine, showed that over 1000 couples wait for 1 to 2 years before undergoing AID treatment (unpublished data). In Beijing, Guangzhou, Nanjing and other large cities across Mainland China, over 10 
000 couples hope to undergo AID as soon as possible. To overcome this challenge of increasing the supply of donor sperm, one method is to permit more than five women to become pregnant using a single donor's sperm. Considering Mainland China's enormous population, the number of donor offspring should be reevaluated. It is suggested that Mainland China carry out the same policy as recommended by ASRM in the United States: limiting a single donor to no more than 25 births would avoid any significant increased risk of inadvertent consanguineous conception.

\section{Minimize the risk of infection and genetics from sperm donors}

There is general agreement among different agencies that sperm donors should undergo rigorous medical evaluation or screening to ensure that no diseases (specifically, sexual or genetic diseases) are passed on to potential offspring. The screening process usually includes taking a medical history from the donor and performing laboratory tests on the semen sample. FIGO recommends that donors of genetic material should be healthy persons of normal reproductive age who are free from sexually transmitted diseases and hereditary disorders. Members of a medical team involved in the management of a gamete recipient should not be donors.

The ASRM 2006 Guidelines for Gamete and Embryo Donation provides guidelines for selection of a donor [11]. The main qualities to seek in selecting a donor for AID are an assurance of good health status and the absence of genetic abnormalities. Although there are no uniformly accepted standards, minimum criteria for normal semen quality can be applied. WHO suggests that several samples be examined before proceeding with a more extensive evaluation [17]. The sample should be examined within $1-2 \mathrm{~h}$ after ejaculation into a sterile container. ASRM publishes minimal semen parameters recommended for donors [18]. The Chinese Ministry of Health has published its own "Screening and Testing Program for Sperm Donors" to establish guidelines for screening and testing of sperm donors. WHO will publish the fifth edition of the Laboratory Manual for the Examination of Human Semen and Sperm-Cervical Mucus Interaction in 2009 with updated semen parameters.

Genetic screening for heritable diseases should also be performed on potential sperm donors. In the United States, testing for cystic fibrosis carrier status is performed on all donors. Other genetic testing should be performed, as indicated by the donor's ethnic background in accordance with current recommendations. Some institutions perform chromosomal analyses on all donors, but such evaluation is not required. In Mainland China, the chromosomal karyotype analysis on all donors is required, but cystic fibrosis carrier status should not be performed on all donors because cystic fibrosis is rare in the Chinese population. Genetic consulting should be performed after screening family history. In addition to adequate history taking and exclusion of individuals at high risk for human immunodeficiency virus and other sexually transmitted infections, laboratory testing should be conducted to ensure that infectious agents will not be transmitted by donor sperm sample [19].

\section{Age requirements for sperm donors}

The United Kingdom, Canada and the United States all specify that sperm donors must be of legal age. In the United Kingdom, HFEA requires that "Gametes should not be taken from anyone under the age of 18 for the treatment of others" [20]. The Canadian ART Act stipulates that sperm or ovum donors must be 18 years old: "No person shall obtain any sperm or ovum from a donor under 18 years of age, or use any sperm or ovum so obtained, except for the purpose of preserving the sperm or ovum or for the purpose of creating a human being that the person reasonably believes will be raised by the donor" [21]. In the United States, ASRM guidelines state: "The donor should be of legal age and, ideally, less than 40 years of age, because increased male age is associated with a progressive increase in the prevalence of aneuploid sperm." The guidelines for selection of anonymous sperm donors in China has been carried out since 2001. Sperm donors should have good health status and no genetic diseases in their family, whose age should be over 22 years and fewer than 45 years, because male aging is associated with a progressive increase in the number of aneuploid sperm. However, as the number of qualified sperm donors is limited for AID or IVF in Mainland China, we recommended that donors' age should be lowered according to ASRM or HFEA. It is important to recruit more donors for sperm banks in China to meet the 
demands from infertile couples.

\section{Anonymous versus non-anonymous sperm donation}

Anonymous versus non-anonymous sperm donation is an important issue to both the recipient and the donor. Sometimes, donors will try to find out who the recipient will be. More often, recipients want to know as much about the donor as possible before undergoing AID. Donor offspring may later inquire about the identity of his or her genetic father as well. A key but long unresolved question in sperm donation is whether the offsprings should be informed of their biological or genetic father and, if so, how much and when the information about donors should be revealed. Parents, donors and offspring may have different interests and views on anonymous versus non-anonymous sperm donation. The practical approach about double track should be considered, which was recommended by ESHRE [4].

\subsection{Anonymous sperm donation}

Those who support anonymous sperm donation insist that anonymity is beneficial to the donor, the recipients and the donor offspring. They express concerns that telling the child of his or her birth by sperm donation will subject the child to social or psychological disorders, which can be especially unsettling if the child wants to find out more information about the donor but cannot. Some studies of children have shown that they are not harmed psychologically by anonymity or nondisclosure, although the children studied may be too young for researchers to draw convincing conclusions $[22,23]$. In addition, anonymous donation allows parents to maintain the issue of infertility as a private matter, which may be vital to them for a variety of reasons. For example, they may be concerned that the child will reject the non-genetic parent, or they may wish to conceal the fact of donation from disapproving family members, especially those from cultures less accepting of sperm donation [24, 25]. In recent years, the question of continued use of sperm from anonymous sperm donors for insemination in couples and the question of insemination of single and lesbian women has been vividly debated. Ernst et al. [26] reported in their survey conducted in Denmark that in 2002, 25\% (19\% approved; 35\% non-approved) of donors stated that they would continue as donors if anonymity was abolished, whereas in 1992 the number was $32 \%$. When donors were asked whether they would accept contact from the children, $22 \%$ agreed in 1992 , but only $13 \%$ agreed (15\% approved; $10 \%$ non-approved) in 2002. From their survey, the authors argued that maintaining anonymity was still important for the vast majority of the donors. Onah et al. [27] investigated the knowledge, attitude and practices of a sample of Nigerian medical students toward sperm donation. With respect to the participants' views on identity disclosure, $35(90 \%)$ of the 39 respondents willing to donate sperm objected to their identities being disclosed to the recipient couples. Although proponents of non-anonymous sperm donation look to the issue of adoption for support for greater disclosure, Patrizio et al. [28] argued that non-anonymous donation distinguishes gamete donation from adoption. They thought it was both wrong and discriminatory to force potential parents to tell their child of his/her genetic origin as a requirement for admission into an AID program. In addition, comparing the practice of using donor semen to conceive a child with adoption was incorrect. Mandatory disclosure was both inappropriate and intrusive, and the presently available data do not justify a rigid position. Furthermore, no reasonable, practical system can be envisaged to guarantee compliance with mandatory disclosure. They argue that, in sperm donation, the rearing mother was usually also the genetic and biological mother, whereas the rearing father was the social father who preferred to keep the donation private.

In the United States, ASRM recommended anonymous or directed (non-anonymous or known) sperm donation [18]. Anonymous donors had traditionally been used, but non-anonymous donation was acceptable if all parties agreed. Directed or non-anonymous donors should undergo the same evaluation as anonymous donors. Both specimens must be quarantined for a minimum of 180 days after donation. The donor must be retested after the required quarantine interval, and specimens may be released only if the results of repeat testing are negative. In Mainland China, only an anonymous sperm donation policy was carried out because traditional Chinese culture or philosophy paid special attention to a child's genetic or biological origin. When parents have children through AID, they prefer to keep this procedure secret from other related parties, including their AID child. It is important to understand that Chinese citizens' have different beliefs on family 
values compared with the Western society, thus there should be continual support for anonymous donation in the future.

\subsection{Non-anonymous sperm donation}

In recent years, a strong tendency in favor of nonanonymous sperm donation has emerged in Europe and Australia. Several countries have enacted laws or are taking into consideration permitting AID children to gain access to information about their genetic fathers. Sweden was the first country to pass legislation about disclosure by establishing a child's right to find out the identity of the gamete donor once the child has reached maturity [29]. The Australian Government published ethical guidelines on the use of ART in clinical practice and research in 2004, which was issued in accordance with the National Health and Medical Research Council Act 1992. People conceived using donated gametes were entitled to know their genetic patents. On request, clinics must arrange for either a medical practitioner, or an appropriately qualified health professional, to provide the donor's information to a person conceived through ART procedures, provided that he or she had either reached the age of 18 or acquired sufficient maturity to appreciate the significance of the request. In 2005, UK legislation was changed requiring any donor of sperm used in AID or IVF to agree to the disclosure of their identity to any offspring reaching the age of 18 [30].

Proponents of non-anonymous sperm donation argued that human beings have a fundamental interest, and perhaps even a legal right, to know their biological origins. Not telling the child of his or her origins violates that child's autonomy. Disclosure was a key part of open and honest communication with children, which helped to avoid secrets in the family that can damage family relationships and generate possible strain and anxieties.

Recent findings [31, 32] showed an increase in donor programs that offer open-identity between donors and offspring. The psychological wishes of sperm donors and their attitudes toward non-anonymity and disclosure are increasingly given consideration. The majority of prospective parents have stated their intention to disclose the method of conception to their children even before the legal changes. Possible influences on intentions included: the culture within the center, movements toward openness within the wider society and parents' lack of confidence regarding how to go about disclosure.
Follow-up studies are needed to improve understanding of whether influences on decision making carry through to patterns of actual disclosure; whether involvement in counseling affects outcomes; and whether access to professional assistance at the time of planned disclosure is helpful. Jadva et al. [33] presented findings from a large sample of donor offspring who are aware of the nature of their conception. Offspring of single mothers and lesbian couples learned of their donor origins earlier than offspring of heterosexual couples. Those told later in life reported more negative feelings regarding their donor conception than those told earlier. Offspring's feelings toward their parents were less clear, with some of those told later reporting more positive feelings and others reporting more negative feelings. Offspring from heterosexual-couple families were more likely to feel angry at being lied to by their mothers than by their fathers. The most common feeling toward fathers was "sympathetic". Age of disclosure is important in determining donor offspring's feelings about their donor conception. It seems that it is less detrimental for children to be told about their donor conception at an early age.

Svanberg et al. [34] had investigated attitudes toward gamete donation among Swedish gynecologists and obstetricians. Among 1230 eligible gynecologists/ obstetricians, $854(69 \%)$ answered the questionnaire. In general, the majority of Swedish gynecologists/ obstetricians had positive attitudes toward gamete donation. Although a majority advocated openness regarding informing the child that he or she was conceived by gamete donation, $\sim 40 \%$ opposed allowing the child to receive any information about the donor when the child has reached maturity. Even though Swedish legislation has allowed sperm donation to lesbian couples since July 2005, one-third of the gynecologists/ obstetricians opposed donation to lesbians. The results indicate that the gynecologists'/obstetricians' negative attitudes toward disclosure may influence patients' ability to discuss their thoughts and feelings about donation. This may also have a negative impact on donor recruitment as well as on the extent of methods made accessible within ART.

Research on how parents of donor offspring make decisions about disclosure reveals that even when couples are initially opposed to disclosing to their offspring, most ultimately come to a united disclosure decision. Shehab et al. [35] had studied how parents whose children have been conceived with donor 
gametes make their disclosure decision. In total, $95 \%$ of couples came to a united disclosure decision, some "intuitively," but most after discussions influenced by the couples' local sociopolitical environment, professional opinion, counseling, religious and cultural background, family relationships, and individual personal, psychological and ethical beliefs. The sperm bank should use an open and consistent approach to ethical issues in the complicated process.

\section{Conclusion and policy suggestions}

This article presents an overview of ethical regulations for sperm donation that are in place from various governmental and non-governmental agencies. From these extensive guidelines, it is clear that the application of sperm donation should concern not only the scientific advances that enable the use of these reproductive technologies but also the ethical considerations and guidelines that should govern sperm donation. For any donor, it is necessary to consider limiting the number of donor offspring, testing the donor and donor sperm for diseases, age requirements and the role of anonymity and non-anonymity. In Mainland China, the anonymous policy for sperm donation should still be carried out, the number of donor offspring should be revaluated, medical standards for sperm donors should be improved according to WHO Laboratory Manual for the Examination of Human Semen and SpermCervical Mucus Interaction, fifth edition, which will be published in 2009.

As developing countries begin to design their own procedures and regulations surrounding reproductive medicine, it will be important to keep in mind these preexisting policies, and to tailor them toward protecting the welfare of all involved parties. It will be necessary to find a balance between both practicality and fairness when designing guidelines for sperm donation. Lastly, sperm donation procedures must be conducted such that the welfare of all participants - the donors, recipients and offspring - are all respected.

Donors must consider (1) why they agreed to help the recipient, (2) how many families or offspring they are willing to help conceive, (3) who will have access to their sperm, (4) what information the offspring should know about the donor, (5) whether they want to be contacted by the recipient or offspring and (6) what they will tell their own children. Recipients must consider (1) whether their partners have agreed to use donor sperm, (2) whether their fertility situation has been properly assessed, (3) what they will tell the child, (4) how much interaction they want the donor to have with the child and (5) what they will do if donation does not work. Both donors and recipients must consider the feelings of the offspring and whether they should know about the donation procedure [16]. As previous research has showed that the offspring benefits from early disclosure [33], it is recommended that the offspring learns about being donor conceived from the recipient couple.

Ultimately, the goal of reproductive medicine is to help infertile couples conceive healthy children. To achieve this end, incorporating all participants' perspectives when formulating ethical procedures and regulations will be both healthy and constructive.

\section{Acknowledgment}

This review was supported by the China National Key Project on the molecular basis of the health of germ cell related to mother/embryo (The ethical principles on development and reproduction, Number 2006CB944010). We appreciate Prof. Yi-Fei Wang, Dr Cappy A. Rothman and Prof. Qing-Li Hu for their critical reading and revisions of our manuscript.

\section{References}

1 Sherman JK, Bunge RG. Effect of glycerol and freezing on some staining reactions of human spermatozoa. Proc Soc Exp Biol Med 1953; 84: 179-80.

2 Critser JK. Current status for semen banking in the USA. Hum Reprod 1998; 13: 55-67.

3 Botchan A, Hauser R, Gamzu R, Yogev L, Paz G, et al. Results of 6139 artificial insemination cycles with donor spermatozoa. Hum Reprod 2001; 16: 2298-304.

4 ESHRE Task Force on Ethics and Law. III. Gamete and embryo donation. Hum Reprod 2002; 17: 1407-8.

5 Weinberg R. The moral complexity of sperm donation. Bioethics 2008; 22: 166-78.

6 Mahlstedt PP, Labounty K, Kennedy WT. The views of adult offspring of sperm donation: essential feedback for the development of ethical guidelines within the practice of assisted reproductive technology in the United States. Fertil Steril 2009 (electronic publication).

7 Hammarberg K, Carmichael M, Tinney L, Mulder A. Gamete donors' and recipients' evaluation of donor counseling: a prospective longitudinal cohort study. Aust NZ J Obstet Gynaecol 2008; 48: 601-6.

8 Ford NM. A Catholic ethical approach to human reproductive technology. Reprod Biomed Online 2008; 
17(Suppl 3): 39-48.

9 Heng BC. Legal and ethical issues in the international transaction of donor sperm and eggs. J Assist Reprod Genet 2007; 24: 107-9.

10 FIGO Committee for the Study of Ethical Aspects of Human Reproduction and Women's Health. Ethical Issues In Obstetrics and Gynecology. London: FIGO House; 2006.

11 Practice Committee of the American Society for Reproductive Medicine; Practice Committee of the Society for Assisted Reproductive Technology. 2006 Guidelines for Gamete and Embryo Donation. Fertil Steril 2006; 86(5 Suppl): S38-50.

12 Human Fertilisation and Embryology Authority. Limitations on the use of gametes (or embryos created using gametes) from an individual donor. HFEA Practice Guidance Note. 2006.

13 Human Fertilisation and Embryology Authority. Who are the donors? An HFEA analysis of donor registrations and use of donor gametes over the last 10 years. 2005 .

14 HFEA Steering Group. Summary of SEED review stakeholder. 26 July 2004.

15 Western Australia Reproductive Technology Council. Questions and answers about the donation of human reproductive material. Western Australia Department of Health; 2005.

16 Melbourne IVF. Sperm donation. The Royal Women's Hospital; 2005.

17 WHO Laboratory Manual for the Examination of Human Semen and Sperm-Cervical Mucus Interaction, 4th edn. New York: Cambridge University Press, 1999.

18 The American Society for Reproductive Medicine. Guidelines for sperm donation. Fertil Steril 2004; 82: S9-12.

19 Center for Biologics Evaluation and Research. Donor screening assays for infectious agents and HIV diagnostic assays. US Food and Drug Administration; 2009.

20 Code of Practice. Human Fertilisation \& Embryology Authority. 2007.

21 Assisted Human Reproduction Act (2004, c. 2). Senate and House of Commons of Canada. 2004.

22 Golombok S. New families, old values: considerations regarding the welfare of the child. Hum Reprod 1999; 13: 2342-7.

23 Amuzu B, Laxova R, Shapiro SS. Pregnancy outcome, health of children, and family adjustment after donor insemination. Obstet Gynecol 1990; 75:899-905.

24 Frith L. Beneath the rhetoric: the role of rights in the practice of non-anonymous gamete donation. Bioethics 2001; 15: 473-84.

25 Mahlstedt PP, Greenfeld DA. Assisted reproductive technology with donor gametes: the need for patient preparation. Fertil Steril 1989; 52: 908-14.

26 Ernst E, Ingerslev HJ, Schou O, Stoltenberg M. Attitudes among sperm donors in 1992 and 2002: a Danish questionnaire survey. Acta Obstet Gynecol Scand 2007; 86: 327-33.

27 Onah HE, Agbata TA, Obi SN. Attitude to sperm donation among medical students in Enugu, South-eastern Nigeria. J Obstet Gynaecol 2008; 28: 96-9.

28 Patrizio P, Mastroianni AC, Mastroianni L. Disclosure to children conceived with donor gametes should be optional. Hum Reprod 2001; 16: 2036-8.

29 Gottlieb C, Lalos O, Lindblad F. Disclosure of donor insemination to the child: the impact of Swedish legislation on couples' attitudes. Hum Reprod 2000; 15: 2052-6.

30 Frith L, Blyth E, Farrand A. UK gamete donors' reflections on the removal of anonymity: implications for recruitment. Hum Reprod 2007; 22: 1675-80.

31 Greenfeld DA. The impact of disclosure on donor gamete participants: donors, intended parents and offspring. Curr Opin Obstet Gynecol 2008; 20: 265-8.

32 Crawshaw M. Prospective parents' intentions regarding disclosure following the removal of donor anonymity. Hum Fertil (Camb) 2008; 11: 95-100.

33 Jadva V, Freeman T, Kramer W, Golombok S. The experiences of adolescents and adults conceived by sperm donation: comparisons by age of disclosure and family type. Hum Reprod 2009; 27; [epub ahead of print].

34 Svanberg AS, Sydsjö G, Selling KE, Lampic C. Attitudes towards gamete donation among Swedish gynaecologists and obstetricians. Hum Reprod 2008; 23: 904-11.

35 Shehab D, Duff J, Pasch LA, Mac Dougall K, Scheib JE, et al. How parents whose children have been conceived with donor gametes make their disclosure decision: contexts, influences, and couple dynamics. Fertil Steril 2008; 89: $179-87$. 